\title{
A Numerical Study on Bubble Detachment from Solid Wall and Formation of Jet inside Detached Bubble
}

\author{
M. U. Afzal and I. S. Kang
}

\begin{abstract}
A numerical study dedicated to observe bubble detachment phenomena from solid wall has been carried out. The Comsol Multiphysics's level set method has been chosen to perform numerical simulations. Numerical results are clearly evidencing bubble detachment and formation of jet inside detached bubble, which after deforming bubble maneuver inside with relatively higher velocity. Detachment time is found to be a direct function of bubble size and shows linear behavior for various fluids. Detailed study reveals that there exists a bubble size effect on jet formation.
\end{abstract}

Index Terms-Bubble detachment, Interface evolution, Level set, Jet, Multiphase flow

\section{INTRODUCTION}

Classically, vapor bubbles are generated from active nucleate sites, growing up to a critical diameter, and then departing from the heating surface, which is so-called nucleate boiling [1]. To understand the complexity and nature of boiling phenomena during nucleate boiling in particular, the knowledge of detachment of vapor bubble from a solid wall is of essential importance. Various attempts have been made during last decades to analyze the dynamics of bubbles and its effects on heat transfer mechanism, but none of them has led to adequate understanding of wholesome process. This might be due to the extreme complex nature of boiling process and involvement of different local transport mechanisms simultaneously. Fast detachment process of bubble from solid wall might increase bubble generation rate and hence consequently heat transfer rate.

In the present work, detachment of gas bubble from horizontal solid wall in addition to formation of jet inside detached bubble are subjects of discussion. This numerical study inscribes the bubble detachment and formation of jet immediately after detachment process and its movement through the bubble for various working fluids. The results seem to be of interest. In our studies, a hemispherical bubble with a fixed contact angle attached to a horizontal solid surface is considered in order to achieve better understanding. Numerous numerical computations have been performed to calculate critical radius of bubble for various fluids that leads to jet formation. As per authors' knowledge, numerical studies on similar observations do not available in the literature.
Manuscript received December 25, 2011; revised February 9, 2012. This work was supported in part by BK21, Korea.

The authors are with the Chemical Engineering Department, POSTECH South Korea (e-mail: umar82088@ postech.ac, iskang@ postech.ac.kr).

\section{Problem STAtement}

In order to study the detachment phenomena, we consider a single gas bubble attached to a solid wall as shown in Fig 1 . Initially, axially hemispherical bubble shape is assumed with $2 / 3 \pi r_{c}{ }^{3}$, where $r_{c}$ is the radius of a bubble sitting on a solid surface and we assumed the contact angle $\theta_{\mathrm{c}}$ of bubble with solid wall is $0^{\circ}$ and imposed perfect wetting condition for the solid wall. The surrounding liquid around the bubble is assumed to be incompressible. To study this problem level set method helps to track interface which moves with fluid particles by solving PDEs like Navier Stoke's equation, continuity equation and level set equation provides a freedom to observe all topological changes. The interface between gas and liquid phase is defined by the level set variable $\phi$, which is a smooth step function that takes the value ' 0 ' in the gas and ' 1 ' in the liquid, with a smooth transition between these values across the interface.

$$
\frac{\partial \phi}{\partial t}+u . \nabla \phi=\gamma \nabla \cdot\left(-\phi(1-\phi) \frac{\nabla \phi}{|\nabla \phi|}+\varepsilon \nabla \phi\right)
$$

The term on the right-hand side of the equation (1) is required to stabilize the equation and to preserve the same smooth profile of $\phi$ across the interface. First term enclosed in left side parentheses represents artificial compression and second term accounts for diffusion. Where ' $\gamma$ ' is the amount of reinitilization and stabilization of the levels set function and ' $\varepsilon$ ' is the interface thickness. Coupling of level set equation with Navier Stoke's and continuity equation is performed to solve the fluid motion and interface evolution in the domain of interest. Effect of surface tension is included in Navier Stoke's equation because of its great physical importance in this problem, this term is introduced to the right side of Navier Stoke's equation

$$
\begin{gathered}
\rho\left(\frac{\partial u}{\partial t}+u \cdot \nabla u\right)-\nabla \cdot\left[\eta\left(\nabla u+\nabla u^{T}\right)\right]+\nabla p=\sigma \kappa n \delta+\rho g \\
\frac{\partial \rho}{\partial \mathrm{t}}+\nabla \cdot(\rho \mathrm{u})=0
\end{gathered}
$$

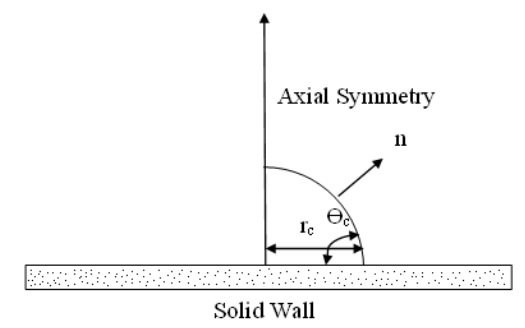

Fig. 1. A hemispherical axial bubble attached to a solid wall 


\section{NUMERICAL SCHEME}

2-D axial geometry for bubble has been drawn in Comsol Multiphysics 3.5 which is a commercial package based on finite element method. First of all, initial solution for the interface has been obtained and stored as 'zero level' for level set function for transient solution of the problem. Physical properties of working fluids are reported in table 1. Suitable values of reinitilization parameter and interface thickness are selected for each case depending on the bubble and mesh size. Boundary conditions are of great importance in numerical computations and total physical understanding of the problem might lead to the proper selection of boundary conditions. Boundary conditions used in this problem are reported in Fig 2. Thinner interface helps to visualize all topological changes which occur across the interface. At time $\mathrm{t}=0$, the velocity and pressure are both zero. The initial $\phi$ is given in fig (3).

\section{RESUlTS AND DisCUSSION}

Numerical simulations of bubble attached to a solid wall has been carried out systematically for R113 (1,1,1-Trichlorotrifluoroethane), Methanol and Ethanol. Sequential images of whole phenomena are reported below in Fig (4): First 3 pictures showing detachment process and after detachment process, and abrupt motion of interface is observed and pictures 6-7 are depicting jet inside bubble which develops with time. At equilibrium a fight between buoyancy and surface tension force starts, when the buoyancy force wins over the surface tension then detachment process starts.

TABLE 1. PHYSICAL PROPERTIES OF WORKING FLUIDS

\begin{tabular}{|c|c|c|c|}
\hline $\begin{array}{c}\text { Working } \\
\text { Fluids/physical } \\
\text { Properties }\end{array}$ & Methanol & Ethanol & R113 \\
\hline $\begin{array}{l}\text { Density, Gas } \\
\left(\mathrm{kg} / \mathrm{m}^{\mathbf{3}}\right)\end{array}$ & 0.31 & 754 & 4.9092 \\
\hline $\begin{array}{c}\text { Viscosity, Gas } \\
\left(\mathrm{Ns} / \mathrm{m}^{2}\right)\end{array}$ & $9.8 \times 10^{-4}$ & $4.52 \times 10^{-4}$ & $9.886 \times 10^{-6}$ \\
\hline $\begin{array}{c}\text { Density, } \\
\text { Liquid }\left(\mathrm{kg} / \mathrm{m}^{3}\right)\end{array}$ & 782 & 789 & 1539.4 \\
\hline $\begin{array}{c}\text { Viscosity, } \\
\text { Liquid (Ns/m²) }\end{array}$ & $5.21 \times 10^{-4}$ & $1.22 \times 10^{-3}$ & $5.733 \times 10^{-4}$ \\
\hline $\begin{array}{c}\text { Surface } \\
\text { tension } \\
\text { coefficient } \\
(\mathbf{N} / \mathbf{m})\end{array}$ & $2.18 \times 10^{-2}$ & $2.41 \times 10^{-2}$ & $1.61 \times 10^{-2}$ \\
\hline
\end{tabular}

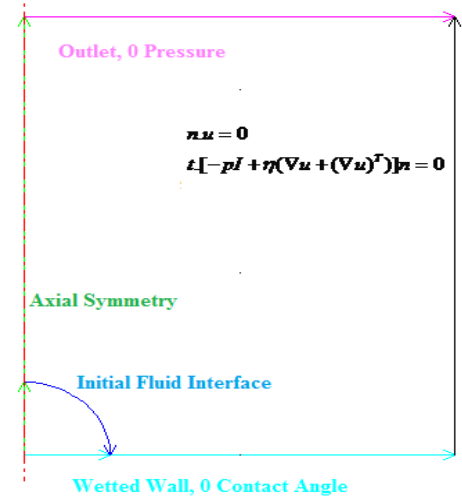

Fig. 2. Details of boundary conditions

In the beginning of jet formation, a much higher velocity
1-1.5 $\mathrm{ms}^{-1}$ has been observed. The thickness of the jet is very small and still requires further investigation to know the effect of bubble size on jet geometry. All these working fluids showed formation of jet inside bubble. In few cases, jet enters inside the bubble from rear end and touches the front end, on the other hand in several cases it remains around the center of the bubble, yet definite reason of highlighted fact is not known. During investigation very interesting observations came up, in some cases a very small sized daughter bubble remains behind as a footprint of mother bubble that segregates from the mother bubble. Fig (6) depicts the highlighted results. Segregation of daughter bubble occurs due to the instability and higher viscous stresses on rear end of bubble. Initially, it seems that there exists a critical region of bubble size which leads to jet formation for various fluids. Velocity field plots supported the jet formation indeed and showed in fig (5). The Laplace-pressure established after the bubble detachment is responsible for the initial acceleration of the liquid from the wall towards the bubble centre. The dynamic effects that immediately follow this movement lead in the case of a smaller bubble to capillary waves along the bubble surface and to oscillations of bubble shape, but in the case of a large bubble, a more or less pronounced jet can form at the rear end of the bubble. This jet formation is to be expected if the inertia of the accelerated liquid is high enough to overcome the counter action of surface tension. For detached bubble, Laplace pressure can be computed by equation 4):

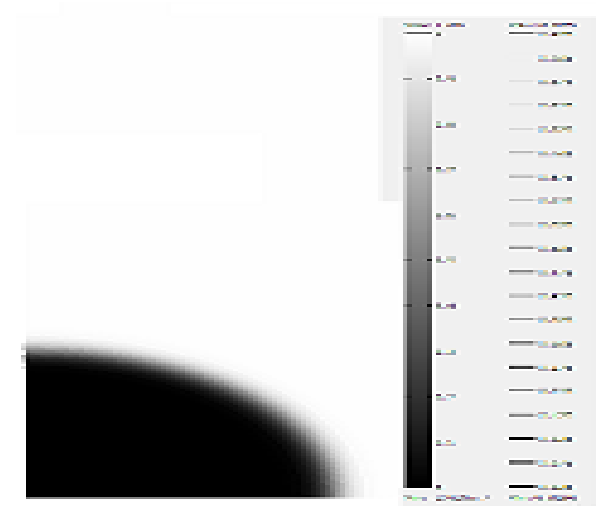

Fig. 3. A surface and contour plot of the initial level set function

$$
\Delta p=p_{v}-p_{L}=2 \frac{\sigma}{r_{0}}
$$

where $\mathrm{r}_{0}$ denotes the radius at the tip just after the bubble detachment and $\mathrm{p}_{\mathrm{v}}$ and $\mathrm{p}_{\mathrm{L}}$ are the pressures at vapor and liquid side respectively. Equation (4) is not applicable at the moment of bubble break-off, because at this moment the radius $r_{0}$ becomes comparable to the size of molecules and the concept of surface tension loses its physical significance. Jet formed inside bubble is continuous and moves upward through the bubble, pierces the bubble surface and leads to considerable, cavitation like 'destruction' [2]. These jet flows happened randomly, and so far no body can exactly tell if jet flow will come forth, or which kind of jets may come forth for specified conditions [3]. The reported numerical results are consistent with the work done by et. al J. Mitrovic., but because of the poor quality of experimental results and unavailability of size information of bubble provided in their work, it seems difficult to make a quantitative and qualitative 
comparison between numerical and their experimental results. Below given fig (7) shows dependency of bubble detachment time on bubble volume and shows a linear behavior, where bubble detachment time is the time required to fully leave contact of bubble with solid wall. Detachment time is solely dependent on physical properties, such as the density and viscosity of both phases and surface tension.

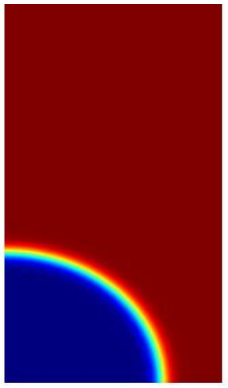

$0 \mathrm{~ms}$

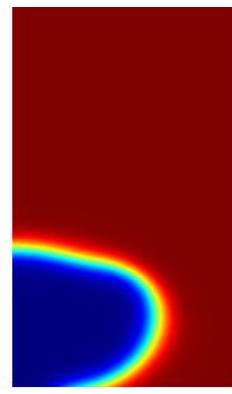

$4 \mathrm{~ms}$

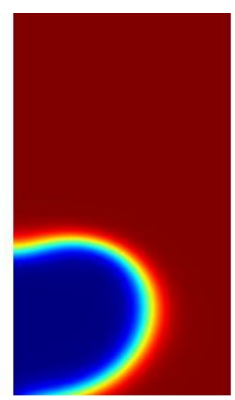

$12 \mathrm{~ms}$

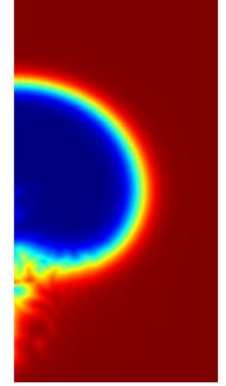

$36 \mathrm{~ms}$

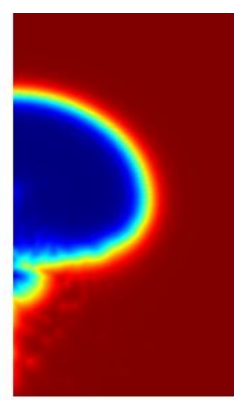

$40 \mathrm{~ms}$

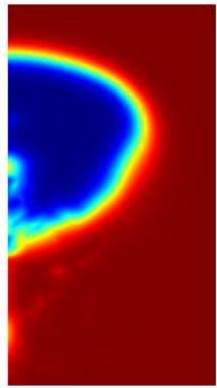

$44 \mathrm{~ms}$

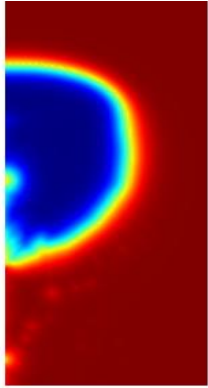

$52 \mathrm{~ms}$

Fig. 4. Ethanol bubble $(2 \mathrm{~mm})$ showing various steps of detachment and jet formation

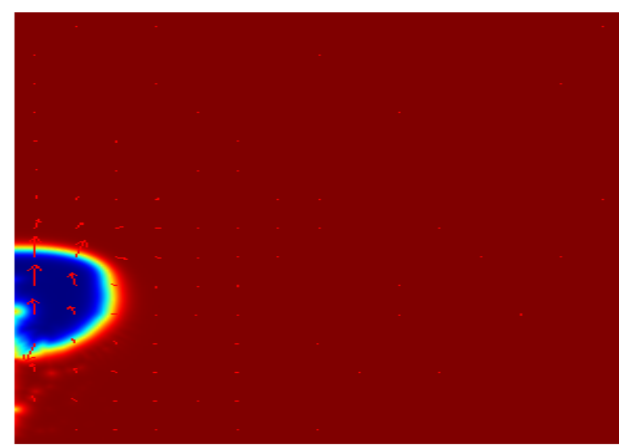

Fig . 5. Velocity field plot for ethanol bubble $(2 \mathrm{~mm})$ at $52 \mathrm{~ms}$, strengthens jet formation

\section{NUMERICAL CHALLENGES}

Level set method faces some numerical challenges like numerical errors due to discretization and estimation of derivates around discontinuities. After detailed investigation of this problem it comes under authors' attention that selection of suitable value of reinitilization parameter is of great importance and assures fast computation and use less physical memory comparatively: and generally its magnitude is of the order of the maximum velocity of the interface. As the bubble volume increases the importance of selection of suitable value of reinitilization parameter and interface thickness becomes more challenging to visualize interface evolutions.

\section{CONCLUSION}

Diversity of bubble detachment was investigated for various fluids and jet formation inside bubble is discussed in detail. From reported numerical results it is clear that bubble detachment time is a linear function of bubble volume, Ethanol bubble shows higher detachment time as compared to R113 and Methanol bubbles because of its greater viscosity and smaller density difference of both phases. Small sized bubbles show almost same detachment time as the volume keeps increasing difference between detachment times among various fluids increased. So far initial investigations gave us a clue about critical bubble diameter for jet formation and depending on the physical properties of

the fluids there will be a critical region for jetting and calculation of critical radius needs further parametric investigation.

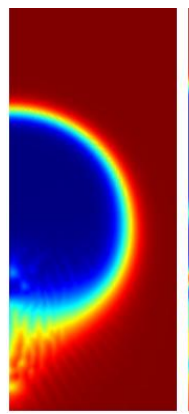
Fig. 6. Methanol bubble (1 $\mathrm{mm})$ showing daughter bubble and jet inside
mother bubble

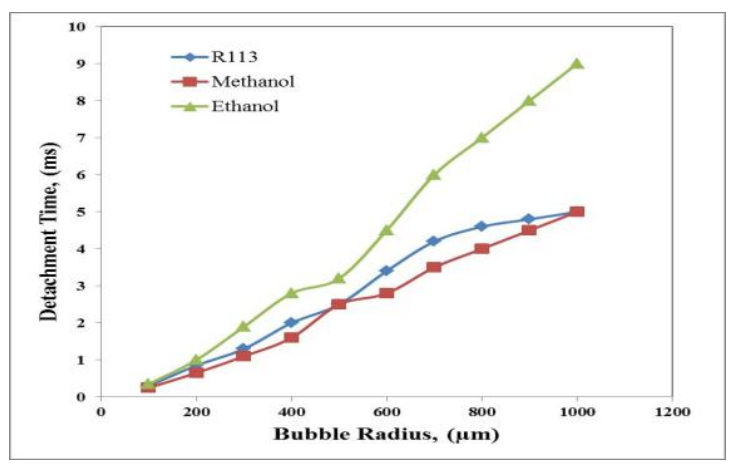

Fig. 7. Relationship of bubble detachment time and bubble radius

Formation of daughter bubble is confirmed by numerical experimentation. This type of jet formation inside bubble might turn to be helpful for mixing of two phases rigorously and hence can be utilized as a potentially new mechanism for various biological and chemical reactions on micro scale fluids.

\section{ACKNOWLEDGMENT}

This research is currently supported by the BK21 Korea.

\section{REFERENCES}

[1] M. Sultan and R. L. Judd, "Spatial distribution of active sites and bubble flux density," ASME J. Heat Transfer, vol. 100, No. 1 pp. 56-62, 1978. 
[2] J. Mitrovic, "Formation of a liquid jet after detachment of a vapour bubble," Int. J. Heat Mass Transfer, vol. 40, No. 18 pp. 4309-4317, March 1997.

[3] H. Wang, X. F. Peng, B. X. Wang, and D. J. Lee, "Bubble sweeping and jet flows during nucleate boiling of subcooled liquids," Int. J. Heat and Mass Transfer, vol. 46, pp. 863-869, 2003

[4] H. J. Cho, I. S. Kang, Y. C. Kweon, and M.H. Kim "Study of the behavior of a bubble attached to a wall in a uniform electric field," Int. J. Mutliphase Flow, vol.22, No.5, pp. 909-922, Jan. 1996.

[5] S.-C. Georgescu, J.-L. Achard, and E.Canot, "Jet drops ejection in bursting gas bubble processes," European J. of Mechanics B/Fluids, vol. 21, pp. 265-280, 2002.

[6] H. Wang, X. F. Peng, B. X. Wang, and D. J. Lee, "Jet flow phenomena during nucleate boiling," Int. J. of Heat and Mass Transfer, vol. 45, pp. 1359-1363, 2002.
[7] L. Duchemin, S. Popinet, and C. josserand "Jet formation in bubbles bursting at a free surface," Physics of fluids, vol. 14, No. 9 pp. 3000-3008, September 2002.

M. U. Afzal was born December 11, 1986 in remote area of Pakistan and got high school education from home town and then shifted to Cultural and knowledge capital of Pakistan Lahore for pursuing Bsc. Chemical Engg from University of the Punjab, Lahore Pakistan. After earning Bsc. Chemical Engg's degree joined M.S. Chemical Engg. Prog in POSTECH, South Korea and since then working in the same university. He worked as an Internee in Irfan Textile industry, Lahore Pakistan for 2 months. Currently working in POSTECH, South Korea as a M.S. student and involved in numerical study of multiphase flows on micro size. Mr. Afzal is a registered memeber of Pakistan Engineering council since from October 2, 2009. 